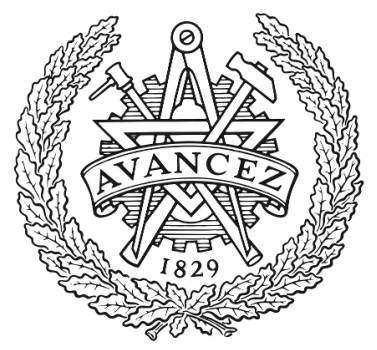

CHALMERS

UNIVERSITY OF TECHNOLOGY

\title{
Intramuscular Stimulation of Muscle Afferents Attains Prolonged Tremor Reduction in Essential Tremor Patients
}

Downloaded from: https://research.chalmers.se, 2023-04-26 04:56 UTC

Citation for the original published paper (version of record):

Pascual-Valdunciel, A., Gonzalez-Sanchez, M., Muceli, S. et al (2021). Intramuscular Stimulation of Muscle Afferents Attains Prolonged Tremor Reduction in Essential

Tremor Patients. IEEE Transactions on Biomedical Engineering, 68(6): 1768-1776.

http://dx.doi.org/10.1109/TBME.2020.3015572

N.B. When citing this work, cite the original published paper.

(O2021 IEEE. Personal use of this material is permitted.

However, permission to reprint/republish this material for advertising or promotional purposes 


\title{
Intramuscular Stimulation of Muscle Afferents Attains Prolonged Tremor Reduction in Essential Tremor Patients
}

\author{
Alejandro Pascual-Valdunciel ${ }^{\mathbb{D}}$, Member, IEEE, Miguel González-Sánchez ${ }^{\mathbb{D}}$, \\ Silvia Muceli ${ }^{\circledR}$, Senior Member, IEEE, Beatriz Adán-Barrientos ${ }^{\circledR}$, Viviana Escobar-Segura ${ }^{(1)}$, \\ Javier Ricardo Pérez-Sánchez ${ }^{\circledR}$, Moon Ki Jung ${ }^{\circledR}$, Andreas Schneider ${ }^{\circledR}$, Klaus-Peter Hoffmann, \\ Juan Camilo Moreno ${ }^{\circledR}$, Senior Member, IEEE, Francisco Grandas, Dario Farina (1), Fellow, IEEE, \\ José Luis Pons, Senior Member, IEEE, and Filipe Oliveira Barroso ${ }^{\circledR}$, Member, IEEE
}

\begin{abstract}
This study proposes and clinically tests intramuscular electrical stimulation below motor threshold to achieve prolonged reduction of wrist flexion/extension tremor in Essential Tremor (ET) patients. The developed system consisted of an intramuscular thin-film electrode
\end{abstract}

Manuscript received June 10, 2020; revised July 29, 2020; accepted August 3, 2020. Date of publication August 19, 2020; date of current version May 20, 2021. This work was supported in part by the European Union's Horizon 2020 research and innovation programme (Project EXTEND-Bidirectional Hyper-Connected Neural System) under Grant 779982 and in part by the Spanish National Research Council, through the Project "Smart" under Grant 202020E121. (Dario Farina, José Luis Pons and Filipe Oliveira Barroso contributed equally to this work.) (Corresponding author: Filipe Oliveira Barroso.)

Alejandro Pascual-Valdunciel is with the Neural Rehabilitation Group, Cajal Institute, Spanish National Research Council (CSIC) and also with the E.T.S. Ingenieros de Telecomunicación, Universidad Politécnica de Madrid.

Miguel González-Sánchez and Javier Ricardo Pérez-Sánchez are with the Movement Disorders Unit, Department of Neurology, Hospital General Universitario Gregorio Marañón.

Silvia Muceli is with the Department of Electrical Engineering Chalmers University of Technology.

Beatriz Adán-Barrientos is with the Bioengineering and Aerospace Engineering Department, Carlos III University of Madrid.

Viviana Escobar-Segura is with the Department of Medicine, Universidad Complutense.

Moon Ki Jung and Dario Farina are with the Department of Bioengineering, Imperial College London.

Andreas Schneider and Klaus-Peter Hoffmann are with the Department of Medical Engineering and Neuroprosthetics, Fraunhofer Institute for Biomedical Engineering IBMT.

Francisco Grandas is with the Movement Disorders Unit, Department of Neurology, Hospital General Universitario Gregorio Marañón and also with the Department of Medicine, Universidad Complutense.

Juan Camilo Moreno is with the Neural Rehabilitation Group, Cajal Institute, Spanish National Research Council (CSIC).

José Luis Pons is with the Neural Rehabilitation Group, Cajal Institute, Spanish National Research Council (CSIC), and the Legs \& Walking AbilityLab, Shirley Ryan AbilityLab, the Department of Biomedical Engineering and Mechanical Engineering, McCormick School of Engineering, Northwestern University, and also with the Department of PM\&R, Feinberg School of Medicine, Northwestern University.

Filipe Oliveira Barroso is with the Neural Rehabilitation Group, Cajal Institute, Spanish National Research Council (CSIC), Madrid 28001, Spain (e-mail: filipe.barroso@cajal.csic.es).

This article has supplementary downloadable material available at https://ieeexplore.ieee.org, provided by the authors.

Digital Object Identifier 10.1109/TBME.2020.3015572 structure that included both stimulation and electromyography (EMG) recording electrodes, and a control algorithm for the timing of intramuscular stimulation based on EMG (closed-loop stimulation). Data were recorded from nine ET patients with wrist flexion/extension tremor recruited from the Gregorio Marañón Hospital (Madrid, Spain). Patients participated in two experimental sessions comprising: 1) sensory stimulation of wrist flexors/extensors via thin-film multichannel intramuscular electrodes; and 2) surface stimulation of the nerves innervating the same target muscles. For each session, four of these patients underwent random 60-s trials of two stimulation strategies for each target muscle: 1) selective and adaptive timely stimulation (SATS) - based on EMG of the antagonist muscle; and 2) continuous stimulation (CON) of target muscles. Two patients underwent SATS stimulation trials alone while the other three underwent CON stimulation trials alone in each session. Kinematics of wrist, elbow, and shoulder, together with clinical scales, were used to assess tremor before, right after, and $24 \mathrm{~h}$ after each session. Intramuscular SATS achieved, on average, $32 \%$ acute (during stimulation) tremor reduction on each trial, while continuous stimulation augmented tremorgenic activity. Furthermore, tremor reduction was significantly higher using intramuscular than surface stimulation. Prolonged reduction of tremor amplitude (24 $\mathrm{h}$ after the experiment) was observed in four patients. These results showed acute and prolonged (24 h) tremor reduction using a minimally invasive neurostimulation technology based on SATS of primary sensory afferents of wrist muscles. This strategy might open the possibility of an alternative therapeutic approach for ET patients.

Index Terms-Essential tremor, Intramuscular, Neuromodulation, Sensory stimulation, Tremor reduction.

\section{INTRODUCTION}

$\mathbf{P}$ ATHOLOGICAL tremor is the most prevalent movement disorder globally and the fundamental clinical sign of Essential Tremor (ET), a medical disorder with an estimated prevalence of $4 \%$ of the population over 65 years [1]. It originates from an aberrant cerebello-thalamo-cortical loop, from which pathological oscillations ultimately project to muscles [2], [3]. The main tremor management approach in ET is medication. First line treatments with established efficacy are propranolol 
TABLE I

SAMPLE DESCRIPTION

\begin{tabular}{|c|c|c|c|c|c|c|c|c|c|c|c|c|c|}
\hline $\mathrm{P}$ & GENDER & AGE & $\mathrm{DD}$ & TREATMENT & $\begin{array}{l}\text { FTM S } \\
\text { (MAA) } \\
{[0-12]}\end{array}$ & $\begin{array}{c}\text { FTM SMT } \\
\text { (MAA) } \\
{[0-20]}\end{array}$ & $\begin{array}{c}\text { FD } \\
{[0-32]}\end{array}$ & $\begin{array}{c}\text { FTM TOTAL } \\
\text { SCORE } \\
{[0-156]} \\
\end{array}$ & $\mathrm{SD}(\%)$ & $\begin{array}{c}\text { CGI-S } \\
{[0-7]}\end{array}$ & ST & IntraStim & SurfStim \\
\hline 1 & $\mathrm{~F}$ & 72 & 7 & PNL & 4 & 10 & 12 & 39 & 40 & 5 & Left & SATS \& CON & SATS \& CON \\
\hline 2 & $\mathrm{~F}$ & 61 & 15 & ZNS, APZ, GBP & 7 & 9 & 8 & 37 & 60 & 4 & Right & SATS \& CON & SATS \& CON \\
\hline 3 & M & 70 & 40 & PMD & 5 & 17 & 13 & 55 & 60 & 5 & Right & SATS \& CON & SATS \& CON \\
\hline 4 & M & 73 & 18 & ZNS, APZ & 5 & 12 & 15 & 46 & 50 & 5 & Left & SATS \& CON & SATS \& CON \\
\hline 5 & M & 63 & 50 & PNL & 5 & 7 & 13 & 36 & 45 & 4 & Right & SATS & SATS \\
\hline 6 & M & 67 & 20 & PNL, PMD & 6 & 7 & 9 & 29 & 70 & 4 & Right & SATS & SATS \\
\hline 7 & $\mathrm{~F}$ & 78 & 40 & APZ, GBP & 5 & 17 & 19 & 62 & 90 & 5 & Right & $\mathrm{CON}$ & $\mathrm{CON}$ \\
\hline 8 & M & 72 & 30 & PMD & 5 & 11 & 14 & 49 & 90 & 5 & Right & $\mathrm{CON}$ & SATS \\
\hline 9 & $\mathrm{~F}$ & 77 & 8 & PNL & 7 & 8 & 13 & 49 & 70 & 5 & Left & $\mathrm{CON}$ & SATS \& CON \\
\hline MEAN & & 70.3 & 25.3 & & 5.4 & 10.8 & 12.8 & 44.6 & 63.8 & 4.6 & & & \\
\hline
\end{tabular}

and primidone [4], although the average reduction of tremor using these medications is only approximately $50 \%$, and one in three patients ends up discontinuing treatment [4], [5]. For these cases, there are second-line therapies, such as deep brain stimulation (DBS) or high-intensity focused ultrasound (HIFU) [6]. DBS requires a highly complex and invasive neurosurgical intervention, while HIFU creates an ablative lesion in the central nervous system, with the impossibility of reversing or regulating its effect. Additionally, both interventions are still highly expensive and cannot be performed in all patients. Thereby, the potential development of less invasive therapies is deemed very appealing. A potential alternative that has been explored is peripheral electrical stimulation.

Some studies have analyzed the effects of surface nerve stimulation using current amplitudes above motor threshold (above MT) to elicit muscle contractions and counteract tremorgenic forces. Two main stimulation strategies have been applied: 1) out-of-phase, which is the most common and generates muscle contraction in the antagonistic muscle to the one presenting tremor activity [7]; and 2) co-contraction, which continuously stimulates a pair of antagonist muscles acting on the same joint to increase its stiffness and, therefore, filter out the tremor [8]. However, fatigue and interference with voluntary movements may prevent the use of stimulation above MT as a therapeutic approach for tremor patients in the long-term.

Recent studies have shown that stimulation of afferent nerve fibers below motor threshold (below MT) may also modulate tremor without the main drawbacks of stimulation above MT [7], [9]. For instance, a wrist-worn peripheral nerve stimulator showed its potential to reduce tremor right after stimulation compared to sham [10], [11]. Another study tested stimulation below MT of several upper limb muscles and reported reduction of wrist tremor during stimulation and also 5 minutes following the end of stimulation [12]. Dideriksen et al. [13] assessed the potential of modulating reciprocal inhibition of wrist flexors/extensors via intramuscular stimulation in two ET patients but results were not conclusive. Important insights on how to improve protocols to apply stimulation below MT have been given by simulation studies: Ia afferents can provide significant common tremor input due to passive stretch, which highlights the interplay between supraspinal input and spinal afferents in tremor generation mechanisms [14]; tremorgenic patterns in ET patients are not constant over time (there can be in-phase and anti-phase activation of antagonists) [15]. Therefore, we hypothesize that selective and timely stimulation of wrist flexors/extensors, guided by continuous electromyography (EMG) recording, will achieve increased levels of tremor reduction in ET patients.

Thereby, following the development of a novel thin-film multichannel electrode [16], the main goal of this study was to test the feasibility of using minimally invasive intramuscular electrodes, guided by adaptive EMG-based algorithms, to reduce wrist tremor in a sample of ET patients. Our second goal was to assess potential prolonged effects of this approach.

\section{Methodology}

\section{A. Participants}

Eleven ET patients were recruited from the Movement Disorders Clinic of Gregorio Marañón Hospital (Madrid, Spain) between April 2019 and January 2020, and were clinically examined by movement disorders specialists of the Neurological Department. Inclusion criteria included diagnosis of ET according to Tremor Research Investigation Group criteria [17], presenting clinically postural tremor; age between 18-80 years; tremor affecting at least one of the upper limbs, with prominent wrist flexion-extension; absence of another neurological or musculoskeletal pathology; ability to understand the procedure and sign the informed consent. Exclusion criteria included coexistence of other diseases that distort movement; mixed or complex tremors, with involvement of multiple muscles and concomitant important medical pathology; and anticoagulant treatment. Two patients were excluded from data analysis because tremor was mostly absent on the first experimental session. Table I reports individual description of the nine patients included in the analyses.

All patients volunteered to participate in this study, were informed about the procedures and possible adverse effects, 
and signed the informed consent to participate. All procedures were conducted in accordance with the Declaration of Helsinki and approved by a local ethical committee, as well as by the Spanish Agency of Medicines and Medical Devices (AEMPS) - record 714/18/EC. Adverse effects were monitored during the experimental session, 30 minutes after, $24 \mathrm{~h}$ after, and one week after each session.

\section{B. Stimulation Strategies}

Two different stimulations were tested in this study.

The first stimulation strategy was selective and adaptive timely stimulation (hereafter denominated SATS). SATS consists on detection of tremorgenic bursts in each muscle and delivery of stimulation pulses to the antagonist when tremorgenic burst overcomes an adaptive threshold. Due to technological limitations, simultaneous stimulation and recording would contaminate EMG data with stimulation artifacts. Thereby, we decided to use sequential operations (recording and stimulation windows), as previously reported [7] (Fig. 1(a)). SATS starts with the analysis of 1s recording window with EMG data from flexor carpi radialis (FCR) and extensor carpi radialis (ECR), and demodulation of both signals to extract the main tremor frequency [18]. If the main frequency falls in the range 3-12 $\mathrm{Hz}$, tremor is detected, an adaptive threshold is updated from the root mean square (RMS) value of the recording window, and the stimulation of the antagonist is enabled for the next $2 \mathrm{~s}$ (stimulation window). Within the $2 \mathrm{~s}$ stimulation window, the RMS of consecutive $10 \mathrm{~ms}$ EMG epochs is computed for both muscles and, if any of the RMS values exceeds its adaptive threshold, a short stimulation burst is delivered to the antagonist muscle (Fig. 1(b)), which allows quasi-synchronous stimulation. Thus, SATS allows not only out-of-phase stimulation of FCR/ECR but also simultaneous stimulation of both muscles if they activate in-phase. The cycle repeats again starting with another $1 \mathrm{~s}$ recording window. The second stimulation strategy was continuous (CON) stimulation. It consisted on consecutive $1 \mathrm{~s}$ recording EMG window followed by $2 \mathrm{~s}$ simultaneous stimulation of both muscles.

\section{Stimulation Sessions}

Each patient underwent two different sessions, which took place at least one week apart and in randomized order: 1) intramuscular stimulation (IntraStim); and 2) surface stimulation (SurfStim). At the beginning of each session, a neurologist assessed the basal condition of each patient by means of the Fahn-Tolosa-Marín [19] tremor rating scale (specific and motor tasks) and the Clinical Global Impression of Severity (CGI-S) / of Change (CGI-C) [20] (pre-CLINIC trial, Fig. 2). Depending on the posture that would elicit increased wrist flexion/extension tremor amplitude, subjects were asked to hold their arms pronated and outstretched, or to hold their elbows flexed and pronated facing both hands, for all trials in the same session. Afterwards, patients were instrumented.

For the IntraStim session, two intramuscular electrodes were inserted in the muscle belly of FCR and ECR of the side most affected by tremor using a hypodermic needle $(25 \mathrm{G}, \mathrm{B}$. Braun

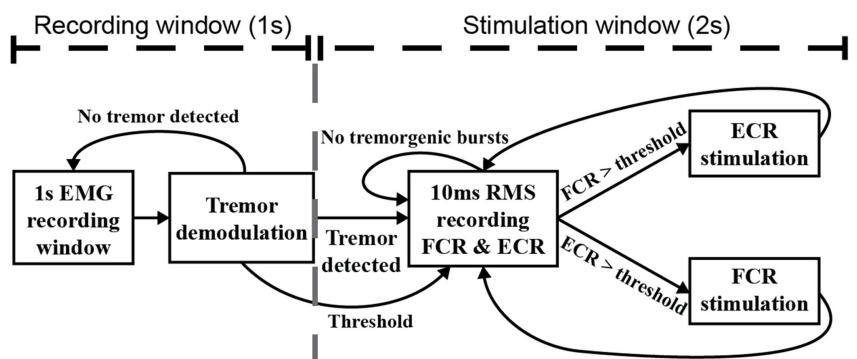

(a)

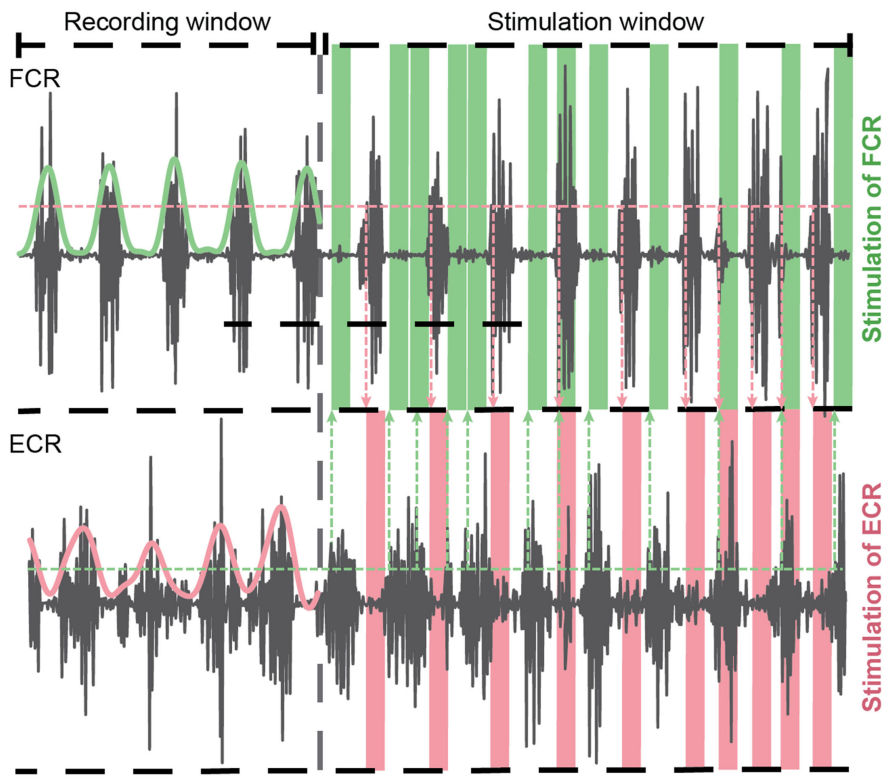

(b)

Fig. 1. (a) Control flow diagram of SATS strategy. (b) Illustration of SATS strategy for a pair of tremorgenic muscles (FCR and ECR). When tremor is detected in the $1 \mathrm{~s}$ recording window, the stimulation is activated for the next $2 \mathrm{~s}$. If tremor is not detected, the recording window restarts. Note that SATS allows simultaneous stimulation of both muscles or consecutive stimulation of the antagonist muscle depending on whether the muscles activated in phase or out-of-phase pattern, respectively.

AG, Germany), under ultrasonography guidance (Supplementary Video 1). The needle was removed after insertion, leaving the electrode within the muscle. These double-sided multichannel electrodes are built on a thin-film substrate of polyimide and embed twelve contacts for EMG recording and three contacts for electrical stimulation (Fig. 3) [16]. EMG data from each of the twelve recording points were visually inspected and the channel presenting qualitatively better signal-to-noise ratio was selected as input for SATS. A customized embedded processing unit that included an EMG amplifier and a voltage-controlled electrical stimulator (OT Bioelettronica, Italy) acquired intramuscular signals at $1,000 \mathrm{~Hz}$. Wet wristbands were used as EMG reference. Additionally, seven inertial measurements units (IMUs) (Technaid S.L., Spain) were placed on each subject: one IMU over the dorsal side of each hand and forearm, one over the lateral side of each arm, and one over the chest. Raw quaternions data from IMUs were sampled at $50 \mathrm{~Hz}$. Finally, a surface electrode $(5 \times 5 \mathrm{~cm}$, ValuTrode Cloth, Axelgaard 
Intramuscular session / Surface session

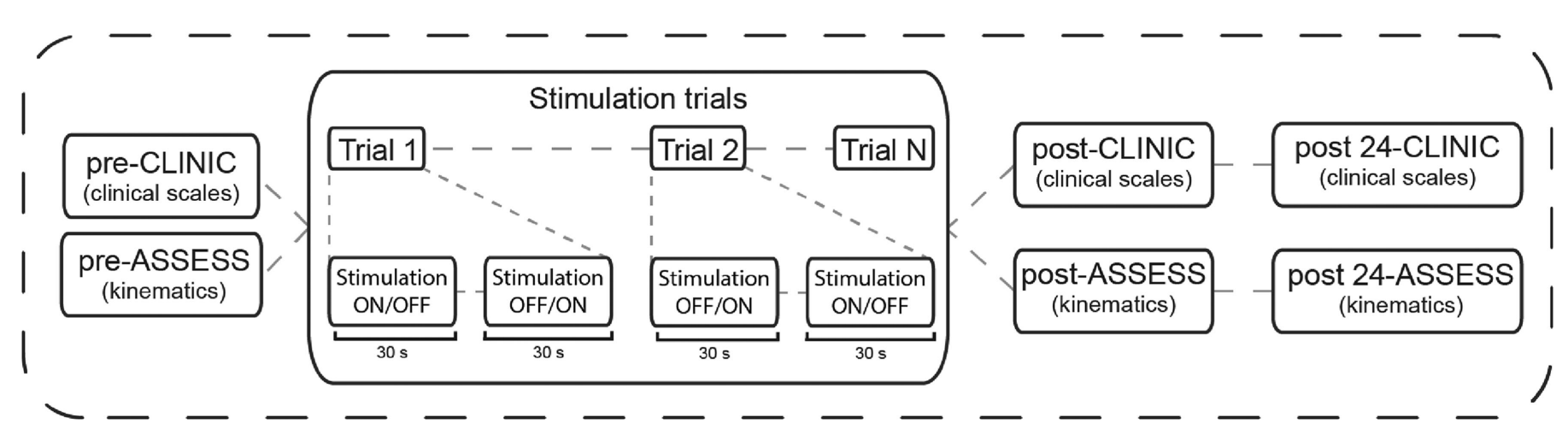

Fig. 2. Schematics of one experimental session for surface (SurfStim) or intramuscular (IntraStim) stimulation. Each stimulation trial lasted $60 \mathrm{~s}$ and was divided in two consecutive $30 \mathrm{~s}$ windows, in which the stimulation system was randomly turned OFF and then ON, or vice-versa. The number of trials completed by each patient $(\mathrm{N})$ depended on the comfort and fatigue perception, ensuring the completion of at least six stimulation trials. Patients were allowed to rest the time they requested between trials.

Top View

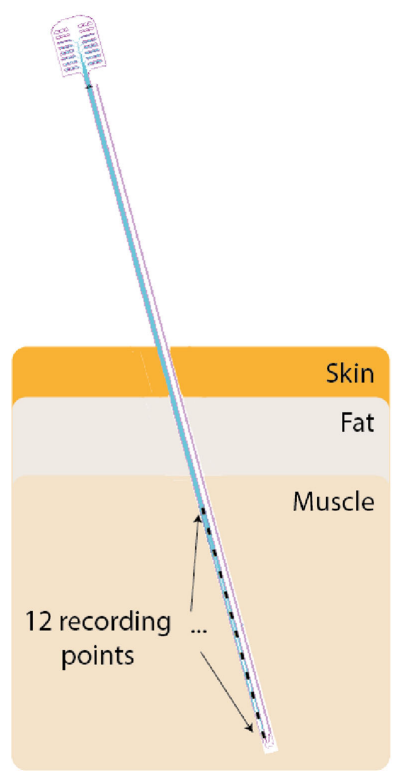

Bottom View

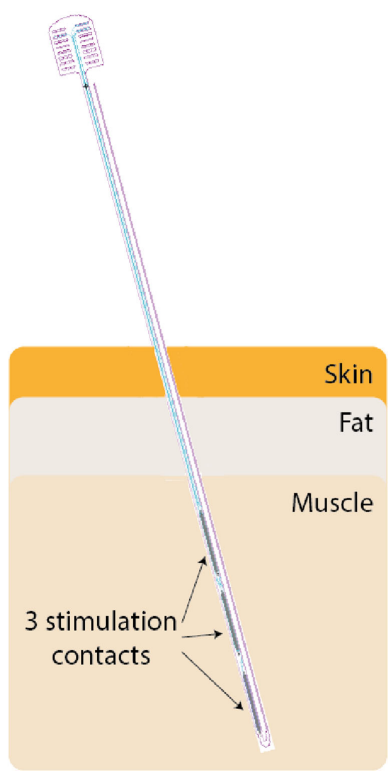

Fig. 3. The thin-film multichannel electrode is made of a polyimide substrate and embeds 12 EMG recording points and 3 stimulation contacts on the two sides of the substrate.

Manufacturing, Denmark) was placed over the olecranon to act as ground for the stimulation.

For the SurfStim session, two round adhesive electrodes (3.2 $\mathrm{cm}$ diameter, ValuTrode Cloth, Axelgaard Manufacturing, Denmark) were placed over the median and radial nerves at the humeral region [21]. A surface ground electrode was also placed over the olecranon. Bipolar surface electromyography (sEMG) electrodes were placed over the muscle belly of FCR (between the medial epicondyle of the humerus and the palmar surface of the base of the second metacarpal) and ECR (between the common head of the lateral epicondyle of the humerus and the base of the third metacarpal), after cleaning the skin with alcohol [22], [23]. Surface EMG signals were acquired at $1,000 \mathrm{~Hz}$. The ground electrodes for EMG recording and stimulation, as well

as the acquisition of the IMU data were unvaried with respect to the IntraStim session.

After instrumenting patients, basal tremor was quantitatively assessed (pre-ASSESS trial): each participant was asked to hold their upper limbs in the same posture as in the pre-CLINIC trial, for $60 \mathrm{~s}$, while IMUs data were recorded. For each session, data from the customized EMG-stimulation unit were synchronized with IMUs data. Raw data were stored and analyzed offline.

After assessing basal condition of tremor, two stimulation parameters were calibrated for each patient and muscle: the perception threshold (PT), which is the current at which the patient started to feel the stimulation, and the motor threshold (MT), defined as the minimum amount of current that elicited motor response. For the IntraStim session, the stimulation amplitude was increased gradually until either the MT or the safety limit of intramuscular electrodes $(2.4 \mathrm{~mA})$ was reached. The deepest of the three stimulation contact points was initially selected for stimulation. When no sensation was evoked through the stimulation of this channel, then the other two channels were used. For the SurfStim session, PT and MT were also identified, and the maximum current delivered never exceeded $5 \mathrm{~mA}$ (technical limitation of the stimulation unit). For both types of stimulation, the maximum current was always below MT and below the safety limit. Stimulation frequency was set at $100 \mathrm{~Hz}$ [13], [24], with biphasic pulse width and maximum stimulation amplitude of $200 \mu$ s and $2.4 \mathrm{~mA}$ for IntraStim [16], and $400 \mu \mathrm{s}$ and $5 \mathrm{~mA}$ for SurfStim [13], respectively.

After calibrating the stimulation parameters, each session proceeded with several stimulation trials, each one consisting of two consecutive $30 \mathrm{~s}$ windows (Fig. 2). For each trial, subjects were asked to hold their arms in the same posture as in pre-ASSESS. In one of the two $30 \mathrm{~s}$ windows, the stimulation unit was turned OFF, whereas SATS or CON stimulation were delivered in the other $30 \mathrm{~s}$ windows (ON). The order of ON and OFF windows for each trial was randomly assigned.

Each patient was blinded to the stimulation strategy applied (SATS or CON). The number of trials completed by each patient depended on the comfort and fatigue perception, ensuring 
the completion of at least six stimulation trials. Patients were allowed to rest the time they requested between trials.

The first four patients enrolled for the study received SATS intermingled with CON stimulation trials, in randomized order, for both sessions. Two other patients underwent SATS stimulation trials alone whereas the other three underwent $\mathrm{CON}$ stimulation trials alone in each session, with the main goal of investigating the independent effect of each stimulation strategy. At the end of each session, the effect of stimulation trials was assessed through kinematics (post-ASSESS) and clinical scales (post-CLINIC) (Fig. 2). A final assessment (post24-ASSESS and post24-CLINIC) was also performed $24 \mathrm{~h}$ after each session to assess possible prolonged effects on tremor reduction.

\section{Data Analysis}

Stored data was analyzed offline using custom software in MATLAB (Mathworks, Natick, MA). Raw data from quaternions were converted into Euler angles and filtered in the tremor band (3ndash;12 Hz) [25], which allowed computation of the angle displacement of wrist, elbow, and shoulder joints. The power spectral density was calculated (2-s Hamming window and $50 \%$ segment overlap) and then integrated in the typical range of tremor frequencies $(3-9 \mathrm{~Hz})$ [13] to quantify the tremor power in the assessments and stimulation trials. For the wrist joints, we calculated the power for the flexion-extension angle, whereas for the elbow and shoulder joints, we calculated the average power for the 2 and 3 degrees of freedom of these joints, respectively. Power values were used to compute different tremor scores, depending on the conditions being compared, according to (1):

$$
\begin{aligned}
\text { Tremor score }= & 0.5+0.5 \\
& \times \frac{(\text { condition } A-\text { condition } B)}{\max (\text { condition } A ; \text { condition } B)}
\end{aligned}
$$

where conditions A and B represent the tremor power or clinical score of two conditions compared through kinematics and clinical scales, such as different assessments, OFF-ON windows (referred as acute tremor score), among others. According to (1), tremor score equal to 1 would correspond to $100 \%$ tremor reduction in condition $\mathrm{B}$ with respect to condition $\mathrm{A}$; tremor score equal to 0.5 would correspond to unchanged tremor amplitude; and tremor score equal to 0 would correspond to $100 \%$ tremor aggravation. Some trials when patients presented very low tremor (power $<0.2 \mathrm{deg}^{2} / \mathrm{Hz}$ ) were excluded from the analysis. Additionally, the trials when patients did not receive SATS stimulation for at least $50 \%$ of the time they should (essentially due to low tremor amplitude) were also discarded from the analysis.

\section{E. Statistical Analysis}

One-sample t-tests were used to compare the effects of each type of stimulation on acute tremor reduction against the tremor score value 0.5 , which corresponds to unchanged tremor amplitude. Independent samples t-tests were applied to compare differences on acute tremor scores between stimulation conditions (SATS IntraStim vs SATS SurfStim, SATS IntraStim vs CON IntraStim, SATS SurfStim vs CON SurfStim) for each joint. Normality tests were applied to check the normal distribution of the data. Statistical significance was set to $p$-value $<$ 0.05 . Descriptive statistics were used to analyze post-ASSESS, post-CLINIC, post24-ASSESS, and post24-CLINICS due to the limited sample size.

\section{REsults}

The number of trials completed and stimulation parameters applied on each patient and session are shown in Table e-1. In some cases, patients did not feel any sensation when stimulation was delivered. For FCR, stimulation amplitude was on average $1.7 \pm 0.8 \mathrm{~mA}$ and $4.8 \pm 0.4 \mathrm{~mA}$ for IntraStim and SurfStim, respectively. For ECR, stimulation amplitude was on average $1.4 \pm 0.9 \mathrm{~mA}$ and $4.6 \pm 0.8 \mathrm{~mA}$ for IntraStim and SurfStim, respectively. On average, $28 \%$ of the completed trials were excluded from the analysis due to very low tremor power at the stimulated wrist.

Information on adverse effects is detailed in Table e-2. Experiments were well tolerated and none of the stimulation sessions caused important adverse events. Seven patients reported transient mild to moderate pain when intramuscular electrodes were inserted or removed. Two patients felt mild transient paresthesias. A patient exhibited moderate fatigue during IntraStim session.

\section{A. Acute Tremor Reduction}

Fig. 4(a) represents wrist flexion-extension angles of a representative patient during pre-ASSESS, during a SATS IntraStim trial, during post-ASSESS, and pos24-ASSESS. Qualitatively, tremor amplitude decreased in post-ASSESS and even more in post24-ASSESS compared to pre-ASSESS, for this patient. Frequency analysis (Y) presented in Fig. 4(b) quantitatively confirmed this observation.

Fig. 4(c) presents mean acute tremor scores for all joints and both sides across all subjects and stimulation conditions. These were calculated according to (1), considering as conditions A and $\mathrm{B}$ tremor power during the $30 \mathrm{~s}$ OFF windows and the $30 \mathrm{~s}$ ON windows, respectively, for each trial and patient. SATS IntraStim was the only condition achieving significant acute tremor reduction $(0.63 \pm 0.03 ; \mathrm{p}<0.01$; equivalent to $26 \%$ tremor reduction $)$ on the stimulated wrist, which was significantly higher compared to the other 3 conditions (SATS SurfStim, CON IntraStim, and CON SurfStim; $\mathrm{p}<0.05$ for the three comparisons). Tremor score was significantly higher $(\mathrm{p}<0.01)$ for SATS SurfStim $(0.53 \pm 0.08)$ than CON SurfStim strategy $(0.35 \pm 0.07)$. In fact, CON stimulation strategy not only did not acutely reduce tremor amplitude at the stimulated wrist, but it even aggravated $(p<0.05)$ tremor during SurfStim.

Although SATS IntraStim achieved, on average, acute tremor reduction on the ipsilateral (stimulated) elbow $(0.60 \pm 0.10)$ and shoulder $(0.58 \pm 0.08)$ (Fig. 4(c)), differences were not significantly higher than $0.5(p=0.06)$. Moreover, there was 




$5 \mathrm{~s}$

(a)

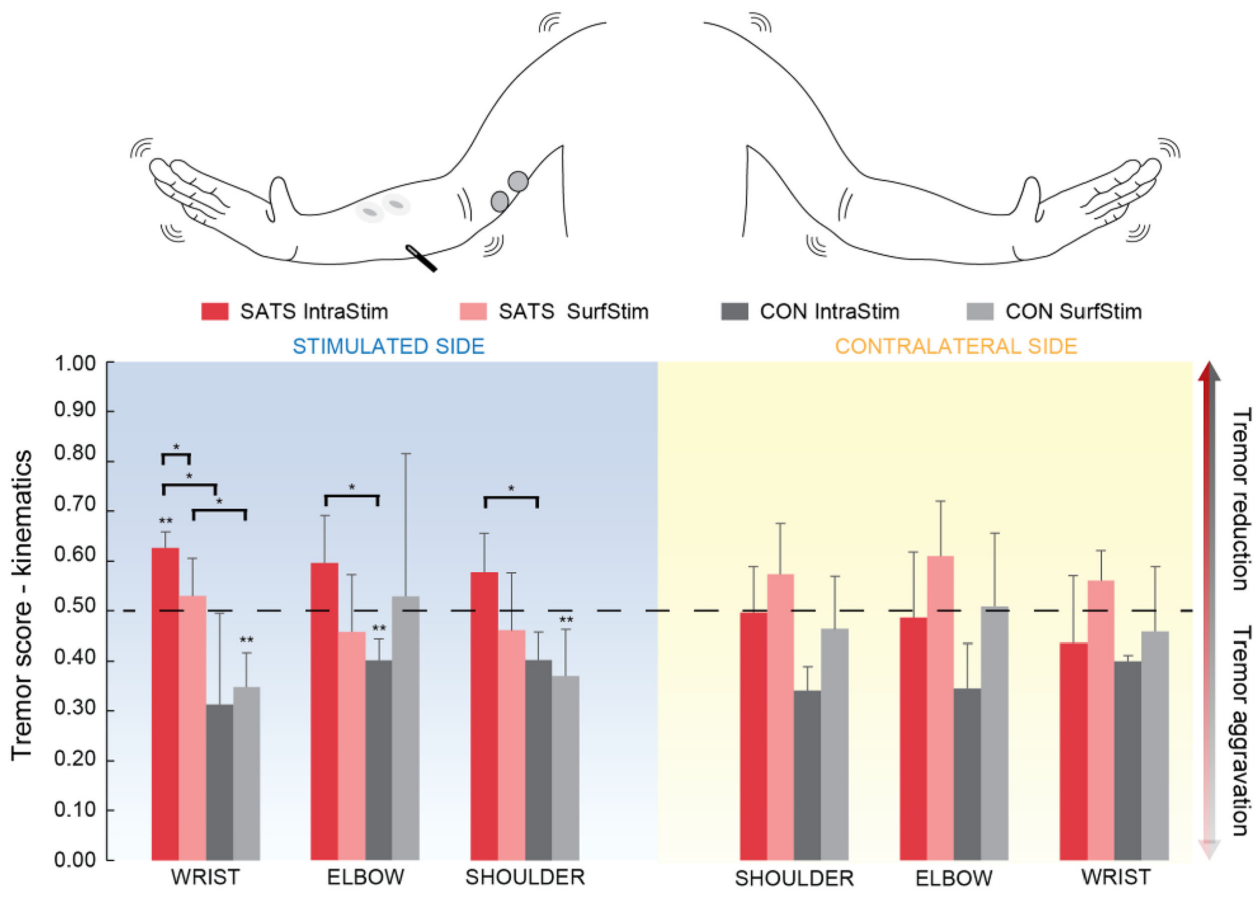

(c)

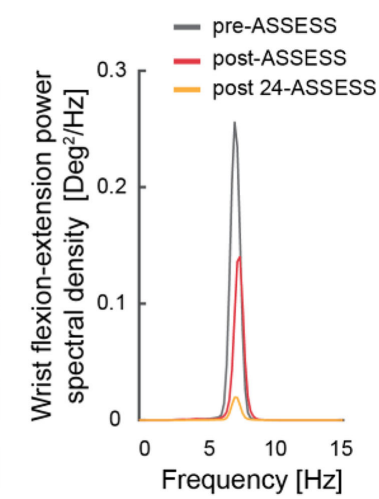

(b)

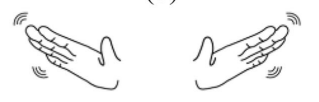

STIMULATION WINDOW

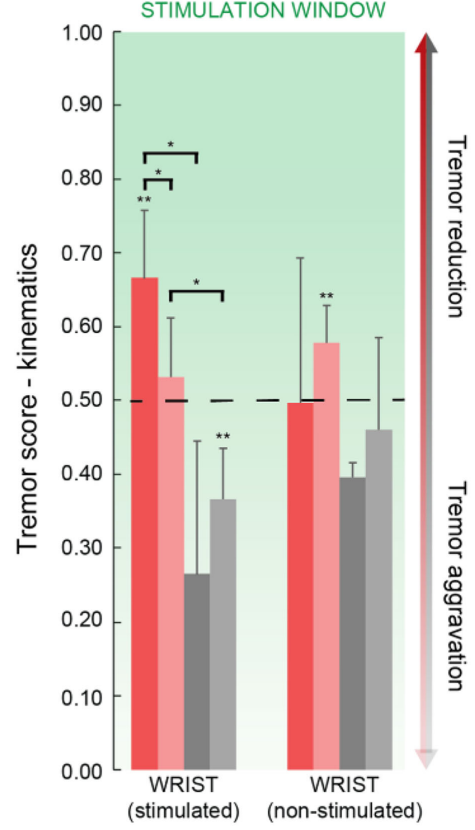

(d)

Fig. 4. (a) Wrist flexion-extension tremor data segments recorded during the IntraStim session of P04. (b) Power Spectral Density of the flexionextension angle of the stimulated wrist for pre-ASSESS, post-ASSESS and post 24-ASSESS for P04 IntraStim session. (c), (d) Acute tremor scores on kinematics for all patients. Results represent mean \pm standard deviation across subjects and trials. (c) Tremor scores between the $30 \mathrm{~s}$ OFF period and $30 \mathrm{~s}$ on period for each assessed joint. (d) Tremor scores between the $30 \mathrm{~s}$ OFF period and the $2 \mathrm{~s}$ stimulation windows segmented from the oN period. Note that tremor score equal to 1 corresponds to $100 \%$ tremor reduction, tremor score equal to 0.5 corresponds to unchanged tremor amplitude, and tremor score equal to 0 corresponds to $100 \%$ tremor aggravation. $* \mathrm{p}<0.05$ (independent samples t-test); $* * \mathrm{p}<0.05$ (one-sample t-test).

no significant acute tremor reduction on any of the contralateral (non-stimulated side) joints.

Fig. 4(d) represents the tremor score as a function of the $2 \mathrm{~s}$ stimulation intervals, with condition A from (1) representing $30 \mathrm{~s}$ OFF windows and condition B from (1) representing $2 \mathrm{~s}$ stimulation intervals inside the $30 \mathrm{~s}$ ON windows from each trial, respectively. In this case, acute tremor reduction on the stimulated wrist was significant for SATS IntraStim strategy ( $0.66 \pm 0.09 ; p<0.02$; equivalent to $32 \%$ tremor reduction), and higher than SATS SurfStim $(0.53 \pm 0.08, p<0.05)$ and CON IntraStim $(0.26 \pm 0.18, p<0.05)$ conditions. SATS SurfStim also achieved acute tremor reduction on the contralateral (non-stimulated) wrist $(0.58 \pm 0.05, p<0.05)$, although differences were not significantly different from the SATS IntraStim condition $(0.50 \pm 0.20)$.

\section{B. Short-Term Effect on Tremor}

Four patients (P01 - P04) underwent random trials of SATS and CON stimulation for both stimulation sessions (IntraStim and SurfStim). Post-ASSESS showed a short-term effect on the stimulated wrist (see Fig. 5(a), Fig. 5(c)). To calculate short-term effects, conditions A and B from (1) were pre-ASSESS and post-ASSESS, respectively. Despite the reduced sample size 


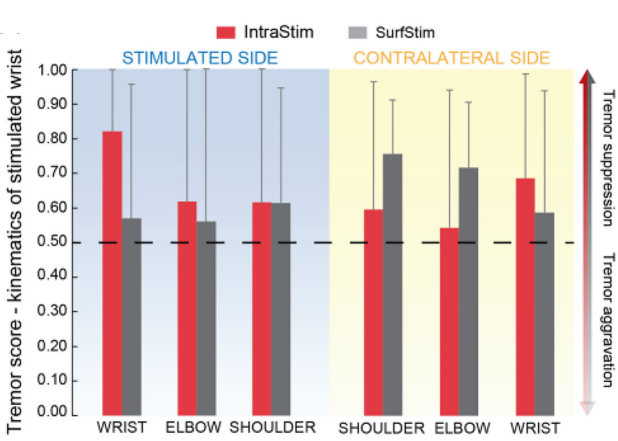

(a)

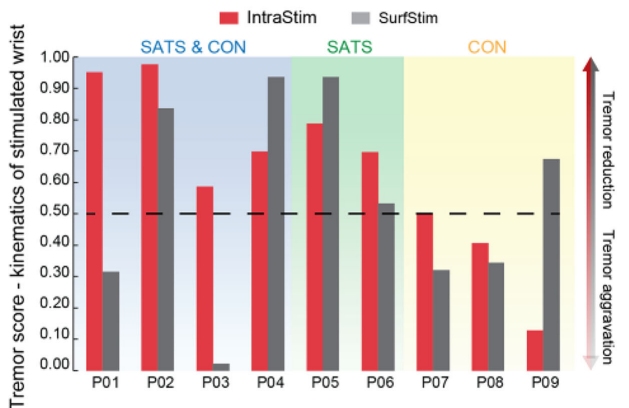

(c)

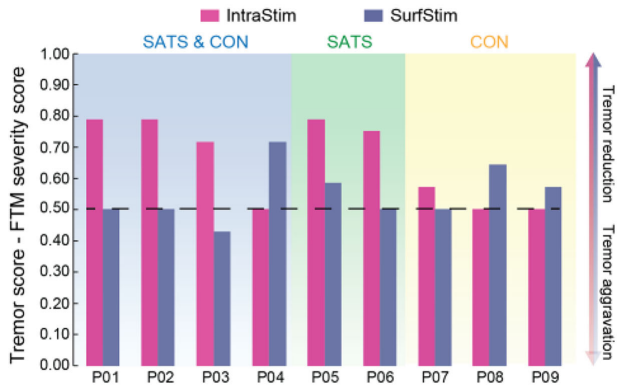

(e)
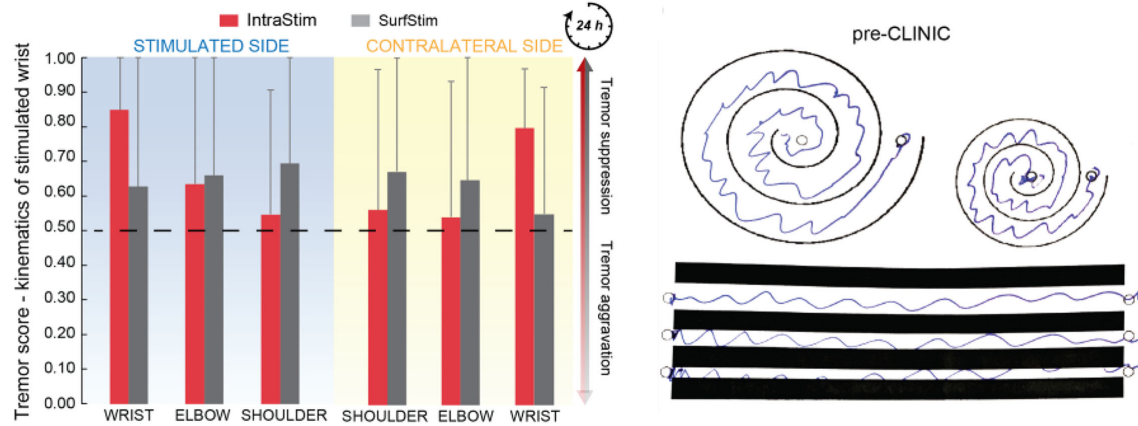

(b)


(d)



(f)

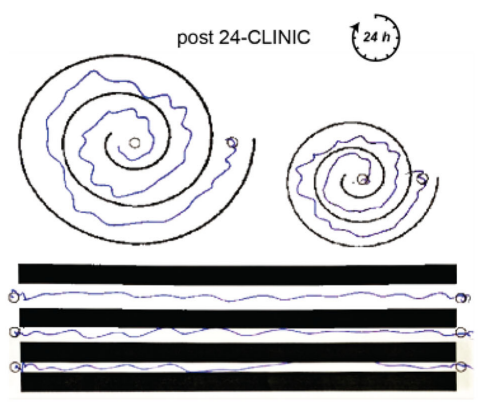

$(\mathrm{g})$

Fig. 5. Short-term (a) (post-ASSESS) and prolonged (b) (post 24-ASSESS) effect of electrical stimulation on the tremor amplitude of several joints, for the first 4 ET patients assessed in the study, who received both SATS and CON strategies. Results represent mean \pm standard deviation of tremor score. Short-term (c) and prolonged (d) effect of electrical stimulation on wrist kinematics tremor score for all patients assessed in the study. Short-term (e) and prolonged (f) effect of electrical stimulation on wrist tremor score, measured by FTM severity scale, for all patients assessed in the study. (g) Spiral drawing item (FTM specific task) performed by P06 in the pre-CLINIC, post-CLINIC and post24-CLINIC during SATS IntraStim session. Spiral drawing are used to clinically assess tremor improvement/worsening. It is possible to notice an improvement (less oscillations in the spiral drawings) during post-CLINIC and post24-CLINIC compared to pre-CLINIC.

( $n=4)$ and variability of tremor, mean reduction of tremor on the stimulated wrist was higher after IntraStim sessions $(0.82$ $\pm 0.20)$ compared to after SurfStim sessions $(0.57 \pm 0.39)$. Nonetheless, tremor score was on average higher than 0.5 at the end of both sessions (post-ASSESS) and all assessed joints (see Fig. 5(a)).

There was also wrist tremor reduction on the two patients (P05 and P06) that only received SATS during the IntraStim session (average tremor score $0.76 \pm 0.07$, Fig. 5(c)), whereas the three patients receiving CON during IntraStim session showed tremor aggravation (average $0.35 \pm 0.2$, Fig. 5(c)). Three patients (P05, P06 and P09) exhibited more prominent tremor on elbow and shoulder joints during the stimulation sessions (see Figure e-1). FTM clinical scale showed that the two patients who received SATS during IntraStim session highly reduced wrist tremor, while there were no tremor changes in those 3 patients that only received CON stimulation (see Fig. 5(e)).

\section{24 h Post Stimulation effect on Tremor}

Tremor reduction was also observed $24 \mathrm{~h}$ after experimentation in 3 out of the 4 patients (P01, P02, and P04) that underwent random trials of SATS and CON stimulation for both stimulation sessions, with an average ipsilateral wrist tremor score of 0.85 \pm 0.22 for the IntraStim session and $0.63 \pm 0.45$ for SurfStim (Fig. 5(b), Fig. 5(d), and results from P02 on Supplementary Video 2). Additionally, tremor reduction was also observed on the contralateral wrist $24 \mathrm{~h}$ after the stimulation for both IntraStim and SurfStim sessions, with mean tremor scores of $0.80 \pm 0.17$ and $0.55 \pm 0.37$, respectively (Fig. 5(b)). 
Regarding the other 5 patients that received SATS or CON stimulation alone, except for one of the 2 patients that received SATS SurfStim, all other patients aggravated tremor 24h after sessions (kinematics results in Fig. 5(d)). However, in general, CON strategy alone aggravated tremor compared to SATS strategy $24 \mathrm{~h}$ after stimulation sessions. According to FTM clinical assessment results, tremor reduction was quite similar in these 5 patients $24 \mathrm{~h}$ after stimulation sessions (Fig. 5(f)). Clinical scales of specific tasks showed that the two patients who received only SATS IntraStim reduced tremor not only after the stimulation (post-CLINIC) session but also $24 \mathrm{~h}$ after session (post24-CLINIC) (Fig. 5(g) and Figure e-2).

\section{DISCUSSION}

This study demonstrates the feasibility of modulating muscle afferents through intramuscular (IntraStim) selective and adaptive timely stimulation (SATS) to decrease tremor. Qualitative results from clinical scales add more evidence to quantitative tremor scores that SATS IntraStim improved voluntary control of daily living tasks and show that this strategy might open the possibility of an alternative therapeutic approach for ET patients. To our knowledge, this is the first report of prolonged (24 h) tremor reduction of wrist flexion/extension in ET patients after delivering intramuscular stimulation below motor threshold for no longer than 10 minutes in total. Short-term effects (right after experimental session) were observed for all six patients who underwent SATS IntraStim trials (P01-P06). We conclude that these effects are not attributed to a placebo effect due to four main reasons: 1) tremor reduction was significantly higher using intramuscular than surface stimulation; 2) SATS IntraStim was the only condition achieving significant acute tremor reduction; 3 ) non-specific (continuous) stimulation usually led to tremor aggravation; 4) patients were blinded to the stimulation strategy applied and, in some cases, they did not feel any sensation when stimulation was delivered.

Other studies have shown the potential of sensory stimulation to reduce tremor in the short-term [7], [10]-[12], [26]. The out-of-phase strategy tested by Dosen et al. [7], which used short recording windows to predict tremor bursts and deliver surface stimulation to the antagonist muscle when the predicted tremor bursts should occur in the agonist, showed good acute results. Those results might be in part explained by reciprocal inhibition due to the out-of-phase stimulation strategy. Dideriksen et al. [13] also assessed the potential of modulating reciprocal inhibition of wrist flexors/extensors via intramuscular stimulation in two ET patients, but results were not conclusive. Our results on acute tremor reduction are robust and demonstrate that reduction was achieved by combining modulation of afferent pathways (low-intensity electrical stimulation of primary muscle spindles) and time-selective stimulation. This selective modulation is hypothesized to drive the inhibitory mechanisms of tremorgenic activity at the spinal cord [21]. The high frequencies and low intensities used here have also been suggested to present several advantages for rehabilitation [27], because synaptic drive from Ia afferents will recruit first smallest, low-threshold, and more fatigue-resistant fibers, which allow delivering more stimulation pulses and, as a consequence, more afferent input [7].

The tremor reduction results observed at the end of IntraStim sessions (short-term effect) are in line with the results reported by others using SurfStim [10]-[12], [28]. All patients who received SATS during IntraStim session (6 out of 6) reduced their tremor during the assessment after the experiment (postASSESS), and some of them held this reduction even $24 \mathrm{~h}$ after the stimulation session (post24-ASSESS). Although prolonged effects are very promising and will be further explored in future studies, in a larger population and also in Parkinson's Disease patients, we can only speculate on the mechanisms underlying these results. We can hypothesize that the prolonged effect induced by electrical stimulation of afferent fibers may be related to plasticity changes at spinal level - perhaps propriospinal pathways - or even at supraspinal levels, modulating central oscillators involved in the pathogenesis of ET. In fact, Tass et al. [29] showed in Parkinsonian monkeys that coordinated reset neuromodulation of the subthalamic nucleus had more pronounced after-effects than classical neuromodulation with DBS-like (high) intensity. Pahwa et al. [11] have also suggested that modulation of afferent pathways may produce an effect in supraspinal centers such as the Ventral Intermediate Nucleus (VIM), which is a brain target for DBS or HIFU for tremor.

In addition to the stimulated wrist, tremor reduction was also observed on the contralateral wrist $24 \mathrm{~h}$ after the stimulation for both IntraStim and SurfStim sessions. A putative explanation could be again the activation of propriospinal interneurons that project bilaterally [30], [31]. Even though SATS IntraStim achieved higher tremor reduction compared to SATS SurfStim, we cannot conclude that surface stimulation should not be explored as an alternative to reduce tremor. In fact, stimulation conditions did not exactly match between intramuscular and surface stimulation due to differences in pulse width, stimulation intensity and electrode placement. Results reported here for SurfStim should be analyzed with caution due to the low stimulation intensity and capability of the stimulator used.

The high cost of thin-film multichannel intramuscular electrodes narrowed the number of patients that could be tested at this stage. Future approaches might include stimulation at multiple joints, especially more proximal ones, because tremor often propagates from proximal to distal joints [32] and this might have an effect in tremor amplitude at more distal joints.

The thin-film intramuscular electrodes used here allow simultaneous stimulation and recording within the same implant. Once improvements in SW/WH that allow concurrent real time EMG recording, artifact removal [33], processing and stimulation emerge, intramuscular electrodes can be developed into fully long-term implantable solutions [34], [35] that will allow tailored tremor reduction through recording and stimulation from/at multiple body parts.

\section{CONCLUSION}

In conclusion, our results show that acute and prolonged (24 h after stimulation) tremor reduction can be achieved in ET patients using our minimally invasive approach based on 
SATS of sensory afferents of wrist muscles. The results of this proof-of-concept study might open the possibility of a new therapeutic approach for tremor patients.

\section{ACKNOWLEDGMENT}

The authors would like to thank Alejandra Garcia Álvarez for her help with data collection and processing, Enrico Merlo and Andrea Bottin from OT Bioelettronica for their assistance on the stimulator unit, and Adriana Muñoz for her help on writing the clinical protocol and preparing necessary documentation for Ethics Committee approval.

Conflicts of interest: JLP, FOB, APV, DF and FG are named inventors on a request for grant of a European patent (submission number 300365138, application number EP20382416.4) that describes a method for controlling a neuroprosthetic device for the treatment of tremors, in particular Essential Tremor and Parkinsons Disease, by intramuscular stimulation of muscle spindles. JLP and DF are named inventors of patent US20140336722A1 that describes a method and neuroprosthetic device for monitoring and suppression of pathological tremors in a user through the neurostimulation of the afferent pathways to the brain.

\section{REFERENCES}

[1] V. Castrillo-Fraile et al., "Tremor control devices for essential tremor: A systematic literature review," Tremor Other Hyperkinet. Mov. (N. Y)., vol. 9, pp. 1-6, 2019.

[2] R. C. Helmich et al., "The pathophysiology of essential tremor and Parkinson's tremor," Curr. Neurol. Neurosci. Rep., vol. 13, no. 9, p. 378, 2013.

[3] E. D. Louis et al., "Essential tremor-plus: A controversial new concept," Lancet Neurol., vol. 19, no. 3, pp. 266-270, 2020.

[4] V.Shanker, "Essential tremor: Diagnosis and management," $B M J$, vol. 366, 2019.

[5] G. Deuschl et al., "Treatment of patients with essential tremor," Lancet Neurol., vol. 10, no. 2. pp. 148-161, Feb. 2011.

[6] J. J. Ferreira et al., "MDS evidence-based review of treatments for essential tremor," Mov. Disord., vol. 34, no. 7, pp. 950-958, Jul. 2019.

[7] S. Dosen et al., "Online tremor suppression using electromyography and low-level electrical stimulation," IEEE Trans. Neural Syst. Rehabil. Eng., vol. 23, no. 3, pp. 385-395, May 2015.

[8] J. Á. Gallego et al., "A neuroprosthesis for tremor management through the control of muscle co-contraction," J. Neuroeng. Rehabil., vol. 10, p. 36, 2013.

[9] F. O. Barroso, A. Pascual-Valdunciel, and J. L. Pons, "Review on tremor suppression using afferent electrical stimulation," in Converging Clinical and Engineering Research on Neurorehabilitation III. ICNR 2018. Biosystems \& Biorobotics., L. Masia, S. Micera, M. Akay, and J. Pons, Eds. New York, NY, USA: Springer, 2018, pp. 1092-1096.

[10] P. T. Lin et al., "Noninvasive neuromodulation in essential tremor demonstrates relief in a Sham-controlled pilot trial," Mov. Disord., vol. 33, no. 7, pp. 1182-1183, 2018.

[11] R. Pahwa et al., "An acute randomized controlled trial of noninvasive peripheral nerve stimulation in essential tremor," Neuromodulation, vol. 22 , no. 5 , pp. $537-545,2019$

[12] J. H. Heo et al., "Sensory electrical stimulation for suppression of postural tremor in patients with essential tremor," Biomed. Mater. Eng., vol. 26, pp. S803-S809, 2015.

[13] J. L. Dideriksen et al., "Electrical stimulation of afferent pathways for the suppression of pathological tremor," Front. Neurosci., vol. 11, Apr. 2017.
[14] J. A. Gallego et al., "Influence of common synaptic input to motor neurons on the neural drive to muscle in essential tremor," J. Neurophysiol, vol. 113, pp. 182-191, 2015

[15] G. Puttaraksa et al., "Voluntary and tremorogenic inputs to motor neuron pools of agonist/antagonist muscles in essential tremor patients," $J$. Neurophysiol., vol. 122, no. 5, pp. 2043-2053, 2019.

[16] S. Muceli et al., "A thin-film multichannel electrode for muscle recording and stimulation in neuroprosthetics applications," J. Neural Eng., vol. 16, no. 2, 2019, Art. no. 026035

[17] K. P. Bhatia et al., "Consensus Statement on the classification of tremors. from the task force on tremor of the International Parkinson and Movement Disorder Society," Mov. Disord., vol. 33, no. 1, pp. 75-87, Jan. 2018.

[18] J. L. Dideriksen et al., "EMG-based characterization of pathological tremor using the iterated Hilbert transform," IEEE Trans. Biomed. Eng., vol. 58, no. 10, pp. 2911-2921, Oct. 2011.

[19] S. Fahn, E. Tolosa, and M. Marín, "Clinical rating scale for tremor," in Parkinson's Disease and Movement Disorders, J. Jankovic and E. Tolosa, Eds. Baltimore-Münich: Urban \& Schwarzenberg, 1988, pp. 271-280.

[20] W. Guy, "Clinical global impressions," in ECDEU Assessment manual for psychopharmacology (Revised) National Institute of Mental Health. W. Guy and. Maryland: Rockville, Ed. 1976, p. 217.

[21] A. Pascual-Valdunciel et al., "Modulation of reciprocal inhibition at the wrist as a neurophysiological correlate of tremor suppression: A pilot healthy subject study," in Proc. 41st Аnnu. Int. Conf. IEEE Eng. Med. Biol. Soc., 2019, pp. 6267-6272.

[22] E. Criswell and J. R. Cram, Cram's Introduction to Surface Electromyography. Sudbury, MA, USA: Jones and Bartlett, 2011.

[23] H. J. Hermens et al., European recommendations for surface electromyography: Results of the SENIAM Project. Roessingh Research and Development, 1999.

[24] J. L. Dideriksen et al., "Physiological recruitment of motor units by highfrequency electrical stimulation of afferent pathways," J. Appl. Physiol., vol. 118 , no. 3, pp. 365-376, 2015

[25] R. J. Elble, "Tremor: Clinical features, pathophysiology, and treatment," Neurol. Clin., vol. 27, no. 3, pp. 679-695, Aug. 2009.

[26] M. Z. Hao et al., "Inhibition of Parkinsonian tremor with cutaneous afferent evoked by transcutaneous electrical nerve stimulation," J. Neuroeng. Rehabil., vol. 14, no. 1, Jul. 2017.

[27] O. Lagerquist and D. F. Collins, "Influence of stimulus pulse width on Mwaves, $\mathrm{H}$-reflexes, and torque during tetanic low-intensity neuromuscular stimulation," Muscle Nerve, vol. 42, no. 6, pp. 886-893, Dec. 2010.

[28] J. Kim, T. Wichmann, O. T. Inan, and S. P. Deweerth, "A wearable system for attenuating essential tremor based on peripheral nerve stimulation," IEEE J. Transl. Eng. Heal. Med., vol. 8, pp. 1-11, 2020

[29] P. Tass et al., "The causal relationship between subcortical local field potential oscillations and Parkinsonian resting tremor," J. Neural Eng., vol. 7, no. 1, pp. 2-3, 2010.

[30] R. Hanajima et al., "Very fast oscillations evoked by median nerve stimulation in the human thalamus and subthalamic nucleus," J. Neurophysiol., vol. 92, no. 6, pp. 3171-3182, 2004.

[31] J. Costa et al., "Subcortical Interactions between somatosensory stimuli of different modalities and their temporal profile," J. Neurophysiol., vol. 100, no. 3, pp. 1610-1621, 2008.

[32] T. H. Corie and S. K. Charles, "Simulated tremor propagation in the upper limb: From muscle activity to joint displacement," J. Biomech. Eng., vol. 141, no. 8, 2019

[33] F. Widjaja, C. Y. Shee, P. Poignet, and W. T. Ang, "FES artifact suppression for real-time tremor compensation," in Proc. IEEE Int. Conf. Rehabil. Robot., 2009, pp. 53-58.

[34] A. Ivorra, L. Becerra-Fajardo, and Q. Castellví, "In vivo demonstration of injectable microstimulators based on charge-balanced rectification of epidermically applied currents," J. Neural Eng., vol. 12, no. 6, Dec. 2015, Art. no. 66010.

[35] L. Becerra-Fajardo, R. Garcia-Arnau, and A. Ivorra, "Injectable stimulators based on rectification of high frequency current bursts: Power efficiency of $2 \mathrm{~mm}$ thick prototypes," in Proc. Converging Clin. Eng. Res. Neurorehabilitation II, 2017, pp. 667-671. 